\title{
MALDI Analysis of Oligonucleotides Directly from Montmorillonite
}

\author{
Dmitri V. Zagorevskii, Michael F. Aldersley, and James P. Ferris \\ Department of Chemistry and Chemical Biology and the New York Center for Studies on the Origin of Life, \\ Rensselaer Polytechnic Institute, Troy, New York, USA
}

\begin{abstract}
Oligonucleotides synthesized on a montmorillonite catalyst were analyzed directly. By mixing the catalyst with a matrix (2,4,6-trihydroxyacetophenone or 6-aza-2-thiothymine) and dibasic ammonium citrate, higher molecular weight products were detected compared with "classical" methods such as gel electrophoresis and HPLC with UV as a detector. The oligomers (30-mers and higher) were detected by mass spectrometry even though their concentration was less than $10^{-4} \%$ of the total content of the RNA. This method is different from the (MALDI) analysis of the eluates from montmorillonite, which otherwise requires desalting. Placing reaction mixtures with a high concentration of buffers on homoionic, preferably Li-containing, montmorillonite does not require desalting. (J Am Soc Mass Spectrom 2006, 17, 1265-1270) (C 2006 American Society for Mass Spectrometry
\end{abstract}

$\mathrm{O}$ ne of the major questions of the origin of life on the Earth is whether oligonucleotides having 40 or more bases could be produced as a result of reactions in the terrestrial environment. We have demonstrated [1-5] that oligomerization of activated nucleic acids can be achieved by using a catalyst, montmorillonite, that occurs naturally on the Earth. Montmorillonite is a clay mineral containing several chemical elements ( $\mathrm{Si}, \mathrm{O}, \mathrm{Na}, \mathrm{K}, \mathrm{Al}, \mathrm{Mg}$ ) and possessing a highly organized layer-type structure [6]. Catalytic oligomerization of a variety of activated nucleotides on montmorillonite has been successfully carried out in our laboratory [1-5]. One of the most common approaches to the analysis of the reaction products involves their extraction from the montmorillonite followed by chromatographic separation and characterization. The latter is usually achieved by gel electrophoresis $[2,4]$ or by the high-performance liquid chromatography (HPLC) method using a UV detector $[2,5]$. This approach is far from ideal because both methods do not provide a "clean" separation of oligonucleotides having similar molecular weights, especially for high molecular weight molecules. Also, the UV detector has a low sensitivity towards large oligomers that are formed in very small quantities/concentrations and may not provide definitive evidence for oligomers of more than $\sim 12$ nucleotides. In the present paper, we are reporting that MALDI-TOF mass spectrometry can be successfully used for the analysis of complex mixtures of oligonucleotides synthesized on montmorillonite without their separation or sample clean-up. The major achievement

Published online June 30, 2006

Address reprint requests to Dr. D. M. Zagorevskii, Department of Chemistry and Chemical Biology, Rensselaer Polytechnic Institute, 209C Cogswell Laboratory, 110 8th St., Troy, NY 12180, USA. E-mail: zagord@rpi.edu of this study is the development of the direct MALDI analysis of the oligonucleotides on the minerals that catalyzed their formation.

\section{Experimental}

\section{Chemicals}

2,4,6-Trihydroxyacetophenone (THAP) and 6-aza-2-thiothymine (ATT) were purchased from Acros Organics (Morris Planes, NJ), and 3-hydroxypicolinic acid (3-HPA) was obtained from Sigma-Aldrich (St. Louis, MO). Sodium perchlorate, acetonitrile, and acetic acid were obtained from Fisher (Fair Lawn, NJ). Dibasic ammonium citrate and ammonium tartrate were purchased from ICN Biomedicals, Inc. (Aurora, OH). MOBS (morpholinobutanesulphonic acid, buffer), TRIS, triethylamine and oligonucleotide standards were bought from Sigma-Aldrich. Montmorillonite was a gift from The American Colloid Company (Arlington Heights, IL). Homoionic montmorillonites (containing lithium, sodium, or potassium) were prepared by the Banin procedure [7]. Water refers to deionized distilled water throughout.

\section{Chemistry}

Adenosine 5'-phosphorimidazolide (ImpA; I) and uridine 5'-phosphorimidazolide (ImpU; II) were synthesized via a published procedure [8]. A typical oligomerization reaction mixture comprised montmorillonite (2 $\mathrm{mg})$ in a solution $(40 \mu \mathrm{L})$ of $0.015 \mathrm{M} \operatorname{ImpA}$ or ImpU, 1 $\mathrm{M}$ alkali metal chloride and $0.1 \mathrm{M}$ MOBS at $\mathrm{pH}=8.0$ and room-temperature for $3 \mathrm{~d}$. The reactions were conducted in filter tubes (Whatman Vecta Spin, $0.2 \mu$ ). By centrifuging for $7 \mathrm{~min}$ at $13.2 \mathrm{k} \mathrm{rpm}$, the montmorillonite was separated from the reaction solution. Ex- 


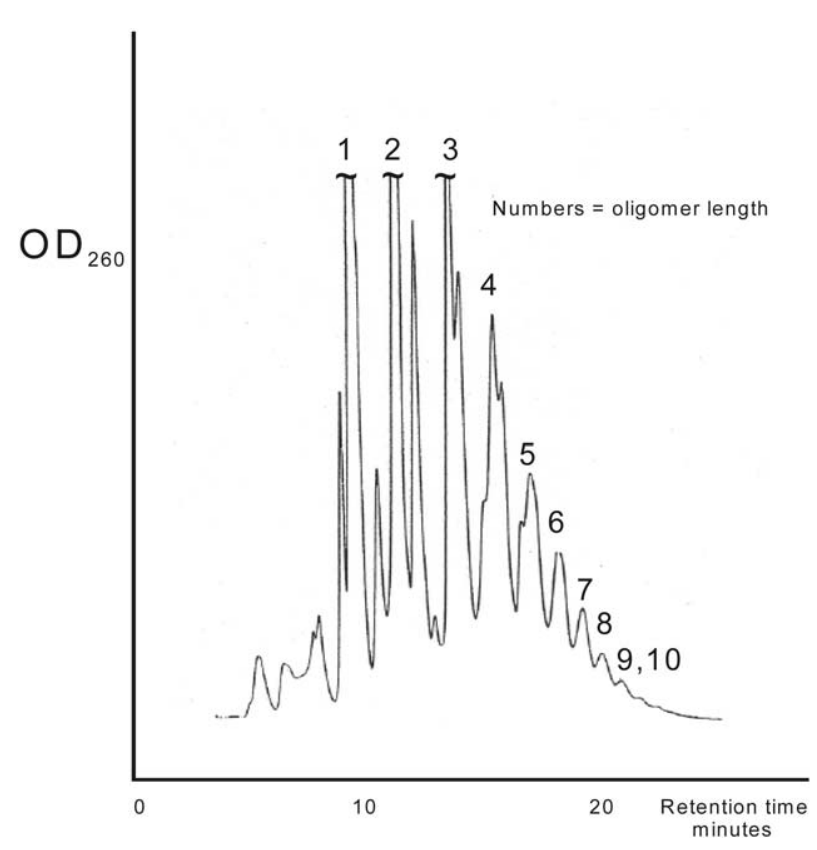

Figure 1. Ion Exchange HPLC analysis of oligomerization products from ImpA on montmorillonite (Sample P). The peak numbers refer to the length of the oligomers.

traction of the solid with $0.1 \mathrm{M}$ alkali metal chloride in $30 \%$ acetonitrile $(40 \mu \mathrm{L})(2 \times 1 \mathrm{~h}, 1 \times 18 \mathrm{~h})$, and further centrifugation provided more product. Ion-exchange HPLC (see Figure 1) was carried out using a Biospher GMB 1000Q column (Puresyn, Inc., Malvern, PA) [3].

\section{I}<smiles>Nc1ncnc2c1ncn2[C@@H]1O[C@H](COP(=O)(O)n2ccnc2)[C@@H](O)[C@H]1O</smiles>

II<smiles>O=c1ccn([C@@H]2O[C@H](COP(=O)(O)n3ccnc3)[C@@H](O)[C@H]2O)c(=O)[nH]1</smiles>

\section{Sample Preparation}

THAP (90 $\mathrm{mg} / \mathrm{mL}$ in methanol) [9], and ATT (10 $\mathrm{mg} / \mathrm{mL}$ in methanol) [10], were tested as matrixes for MALDI. 3-HPA is recommended for the analyses of high molecular weight oligonucleotides [11], but no signal was observed when this compound was used as a matrix in our experiments. This fact was consistent with the observations of other investigators [12]. Both THAP and ATT produced meaningful signals of the product oligonucleotides. THAP was the preferable matrix because it provided better detection of higher molecular weight products as compared with ATT. Dibasic ammonium citrate was a better signal enhancer than diammonium tartrate or ammonium acetate in these experiments.

Oligomers formed by montmorillonite catalysis were analyzed directly on the catalyst surface by combining $\sim 2 \mathrm{mg}$ of the mineral with $5 \mu \mathrm{L}$ of the THAP matrix and $2 \mu \mathrm{L}$ of $0.5 \mathrm{M}$ ammonium citrate; all the components were thoroughly mixed and applied to the MALDI plate using a stainless steel spatula.

In the experiments with homoionic montmorillonites as comatrix, $10 \mu \mathrm{L}$ of the reaction extract was mixed with $\sim 2 \mathrm{mg}$ of the dry mineral, which had been combined with $5 \mu \mathrm{L}$ of the THAP matrix and $2 \mu \mathrm{L}$ of $0.5 \mathrm{M}$ ammonium salt, mixed to produce a homogenous paste, which than was applied to the MALDI plate. It is recommended that the montmorillonite be wetted before mixing with the other components, since the dry montmorillonite can have difficult handling properties because of electrostatic effects.

Desalting of reaction solutions was performed using $\mathrm{C}_{18}$-ZipTips (Brinkman Instruments/Eppendorf, Westbury, NY). Triethylammonium acetate (synthesized by direct neutralization of triethylamine with acetic acid, followed by freeze drying) and water were used consequently to remove salts/buffers from RNA absorbed on the $\mathrm{C}_{18}$-stationary phase. Oligonucleotides were removed from the ZipTips by a water-acetonitrile (50:50) solution.

\section{Mass Spectrometry}

MALDI mass spectra were recorded using a Micromass TofSpec-2E mass spectrometer operated by MassLynx software, (version 3.5, Wythenshawe, UK) in reflectron and linear modes. The detection of high molecular weight products was performed in linear mode by turning on the high mass detector $(7-9 \mathrm{kV})$. A laser pulse voltage of $3500 \mathrm{~V}$ was applied to improve the detection of larger molecules. The energy of the laser was set for the highest yield of the ions by optimizing coarse and fine laser powers. These values were 50 and $80 \%$, respectively, for montmorillonite samples, and 20 and $80 \%$, respectively, for desalted samples. The power of the laser before the laser beam passed through all lenses was $\sim 160 \mu \mathrm{J}$. 
Both negative and positive ion modes could be used for the analyses of the mixtures of oligonucleotides. MALDI(+) mass spectra showed better resolution than MALDI(-) mass spectra in linear mode at the same laser powers. However, the detection of negatively charged ions provided better sensitivity for the high molecular weight oligomers. The highest mass oligomers detected in negative ion mode were 1.5-2 times larger than those identified by MALDI(+). Also, MALDI(-) mass spectra were less complicated and contained fewer peaks than the corresponding MALDI(+) mass spectra.

Calibration of the mass scale in the negative ion mode was performed using clusters of MOBS and a standard mixture of adenosine oligonucleotides. A mixture of peptides was used for the calibration in positive ion mode.

\section{Results and Discussion}

Mass spectrometry provides a variety of tools for the detection and identification of DNA and RNA [13-15]. MALDI is one of the most convenient ionization techniques for the analysis of individual oligonucleotides and their mixtures. This technique, however, generally requires at least a partial purification of the oligonucleotides [13]. The presence of buffers may substantially diminish or completely eliminate signals due to the analyte(s) in MALDI mass spectra. In such cases, a clean-up of the sample is required. In the present work, we report the results of the analyses of complex mixtures of oligonucleotides synthesized on montmorillonite in the presence of salts and buffers. The applications of homoionic montmorillonites for molecular weight determination and structural analyses of DNA/RNA will be discussed.

Several types of samples obtained from the reactions described in the Experimental section were subjected to

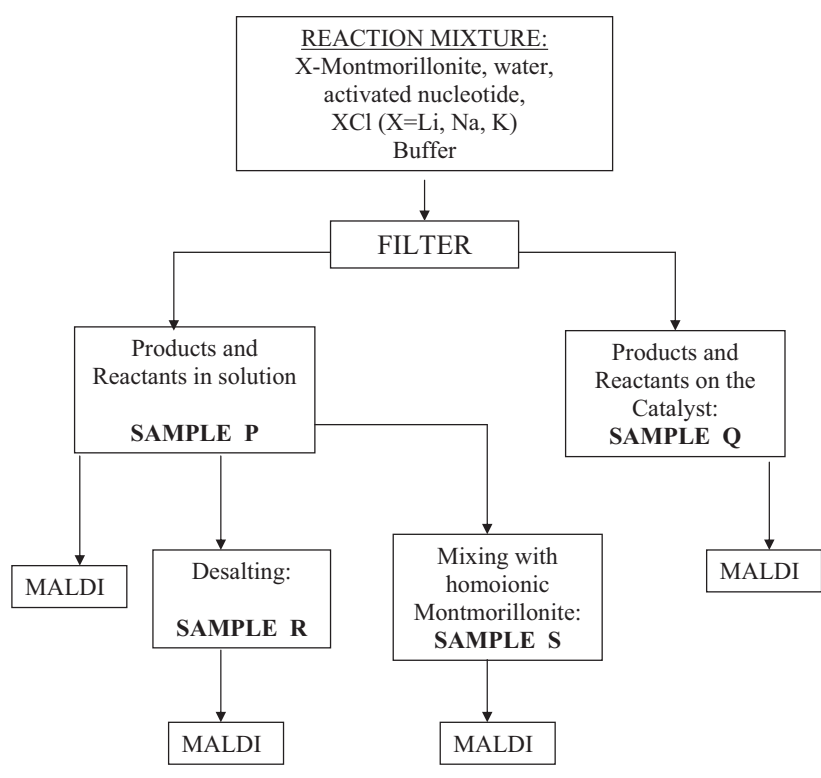

Scheme 1
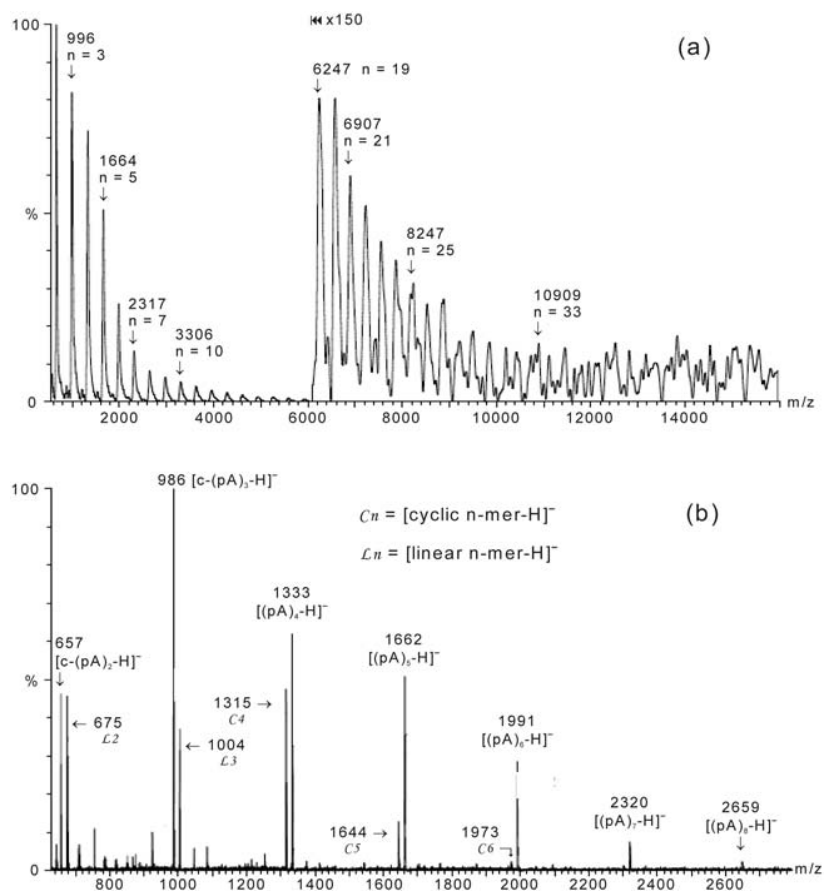

Figure 2. (a) Linear and (b) reflectron mode MALDI( $(-)$ mass spectra of the mixture of oligomerization products from ImpA on montmorillonite shown on Figure 1 after desalting.

MALDI analysis. A simplified diagram of a typical chemical experiment and reaction components are shown in Scheme 1. Two major parts of the mixture were the solution (Sample P) and the montmorillonite (Sample Q). Both contain the oligomerization products, but only the Sample P has been subjected to chemical analyses in previous studies [1-5].

We performed MALDI analysis on products in Sample $\mathrm{P}$ without further purification. The majority of these analyses were unsuccessful. Such a result was somewhat predictable and was caused by the high concentrations of alkali metal chloride and buffer in the samples. MALDI mass spectra showed very distinctive signals of the buffer clusters, [(MOBS $-\mathrm{H}+\mathrm{Na})_{n}-$ $\mathrm{Na}^{-}$, separated by $245 \mathrm{Da}$, (MOBS $-\mathrm{H}+\mathrm{Na}$ ). No signals due to oligonucleotides were observed even though the examination of the samples by the HPLC/UV method (Figure 1) provided unequivocal proof of their presence.

The reaction mixtures in Sample $\mathrm{P}$ were desalted with $\mathrm{C}_{18}$-ZipTips and the mixtures (Samples $\mathrm{R}$ ) were subjected to MALDI analyses. As expected, desalting resulted in a great improvement in the quality of the mass spectra. Figure 2 displays a MALDI(-) mass spectrum of the desalted mixture of oligonucleotides from ImpA. The reflectron mode MALDI mass spectrum displayed several families of peaks separated by 329 Da due to a (pA) unit. Two series of ions dominated the mass spectrum; these series correspond to deprotonated linear, $(\mathrm{pA})_{\mathrm{n}}$ and cyclic, $\mathrm{c}-(\mathrm{pA})_{\mathrm{n}}$ products, with a characteristic mass difference of 18 Da. (A pure linear 
oligomer never gives the cyclic counterpart under our MALDI MS conditions.)

The analysis of the Samples R in linear mode (Figure 2) allowed for detection of oligomers containing more than 30 nucleotides. It should be noted that high molecular weight oligonucleotides have generally lower probability of detection by matrix-assisted laser desorption ionization than their smaller, lower molecular weight analogs [13]. The reasons for this effect are a higher susceptibility of larger oligonucleotides to decomposition under MALDI conditions and their poorer desorption from the MALDI matrix. According to the HPLC analyses and to the reaction kinetics modeling, the probability of the formation of oligomers rapidly decreases with their size. For example, the evaluated concentration of dodecamer should not be higher than $0.01 \%$ of the concentration of decamer but the ratio of peak intensities in the MALDI mass spectrum (Figure 2a) did not differ by more than 20 times. The intensity distribution of peaks due to various oligomers in the MALDI mass spectra seems to be in contradiction with their expected relative yields. The following factors are likely contributors to the increased sensitivity to the larger oligomers. First, from the reflectron mode data it was apparent that there was no detectable dissociation of oligomers up to ten nucleotides, such as loss of water, base, etc. [13]. Only very scarce post-source dissociation was observed at the used laser energies. Second, in our experiments the mass spectrometer was set up for the highest yield of heavy ions and for their preferable detection. This was achieved by tuning the laser pulse duration and laser energy, and by using the high mass detector, respectively. Another likely reason for the enhanced detection of large oligonucleotides is that their smaller analogs are partially washed from the $\mathrm{C}_{18}$-stationary phase of the ZipTips during the desalting procedure. These factors resulted in the sensitivity to the larger oligonucleotides being comparable to or even higher than the sensitivity to their smaller analogs thus making MALDI as a very useful and reliable method for the analyses of these mixtures.

One of the major concerns for analyses of Samples $\mathrm{P}$ by non-mass spectrometric methods is whether oligomers extracted from the montmorillonite represented all the products formed in the course of the reaction. There is a possibility that molecules of principal interest, i.e., those having the highest molecular weight, remain on the mineral catalyst. Also, they could remain on the membranes used for filtering the reaction solutions. To resolve this problem, direct MALDI analyses of the montmorillonite that had been used as the catalyst (Samples Q) were performed. Catalyst samples were mixed with a matrix and an ammonium salt as described in the Experimental section. Linear and reflectron mode MALDI mass spectra of the catalyst containing the products of oligomerization of ImpA are shown on Figure 3.

The mass spectrum from the material on the Namontmorillonite catalyst (Figure 3a) displayed peaks of

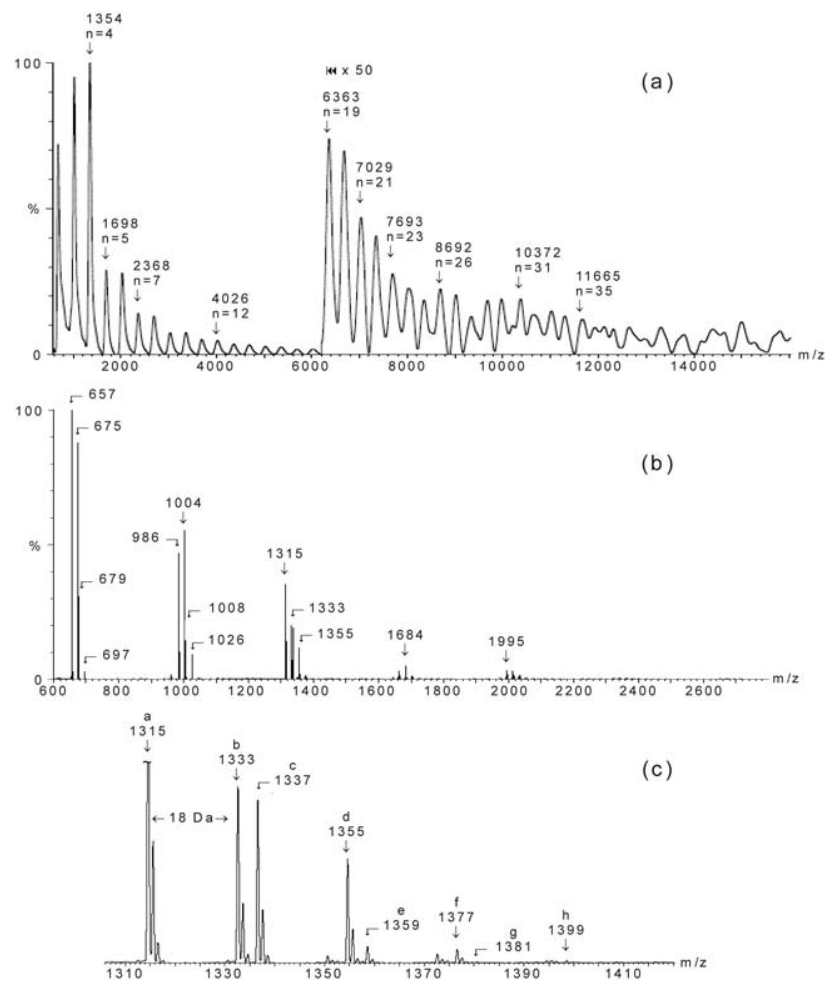

Figure 3. Direct MALDI(-) analysis of the montmorillonite (sample Q); ImpA oligomerization in (a) linear and (b) reflectron modes; (c) is an enlargement of the region containing tetramer ions. Peaks marked with letters correspond to the following ions: $\mathrm{a}\left[\mathrm{c}-(\mathrm{pA})_{4}-\mathrm{H}\right]^{-}, \mathrm{b}\left[(\mathrm{pA})_{4}-\mathrm{H}\right]^{-}, \mathrm{c}\left[\mathrm{c}-(\mathrm{pA})_{4}+\mathrm{Na}-2 \mathrm{H}\right]^{-}, \mathrm{d}\left[(\mathrm{pA})_{4}\right.$ $+\mathrm{Na}-2 \mathrm{H}]^{-}$, e $\left[\mathrm{c}-(\mathrm{pA})_{4}+2 \mathrm{Na}-3 \mathrm{H}\right]^{-}, \mathrm{f}\left[(\mathrm{pA})_{4}+2 \mathrm{Na}-3 \mathrm{H}\right]^{-}, \mathrm{g}$ $\left[\mathrm{c}-(\mathrm{pA})_{4}+3 \mathrm{Na}-4 \mathrm{H}\right]^{-}, \mathrm{h}\left[(\mathrm{pA})_{4}+3 \mathrm{Na}-4 \mathrm{H}\right]^{-}$.

oligonucleotides. The difference between the signals was $\sim 329$ Da corresponding to the pA unit. The size of RNA oligomers detected directly from the montmorillonite was approximately the same as that observed for the desalted reaction solution (Figure 2a). Unlike the unpurified solution (Sample P) and similar to the desalted Sample $R$, the MALDI mass spectrum of the Sample Q showed no signals attributable to the buffer clusters. Several conclusions can be drawn based on the comparison of these mass spectra. Most importantly, the extraction procedure is indeed removing the longest oligomers from montmorillonite since there is no significant difference in the detected masses of oligomers in Samples Q and R. Either sample is appropriate for monitoring these reaction systems. To observe MALDI mass spectra from the montmorillonite, higher laser energies were required. This may be a source for additional dissociation of oligomers and results in the disappearance of the signals due to the highest mass molecules.

Peaks in linear mode MALDI mass spectra of samples where montmorillonite was the "comatrix" were wider than those observed for the desalted samples. This was caused by the presence of alkali metals ( $\mathrm{Na}$ and $\mathrm{K}$ ) in montmorillonite, which resulted in multiple metal-hydrogen exchanges and broadening the signals 
(Figure 3a) compared with the desalted sample. The presence of the $\mathrm{H} / \mathrm{Na}$-exchange products is clearly indicated by the MALDI mass spectrum of the Sample $\mathrm{Q}$ recorded in reflectron mode (Figure $3 \mathrm{~b}$ and $\mathrm{c}$ ). As a result, it can be expected that the analyses of the samples of montmorillonite will have lower sensitivity compared with the analyses of the corresponding desalted samples. At the same time, the incorporation of alkali metals into oligonucleotides is expected to increase the stability of their ions [16, 17]. In spite of the disadvantages outlined, the direct MALDI analyses of the montmorillonite should be considered as an acceptable technique for the analysis of oligomerization products of RNA on montmorillonites. The large difference between the mass spectra of the catalyst and original, unpurified Sample $\mathrm{P}$ is that the former showed no buffer peaks, i.e., no special clean up procedure is required for catalyst samples. A simple mixing with a matrix and an ammonium salt resulted in the observation of a high quality mass spectrum, which displayed all the characteristics of the desalted Sample R.

The exact reasons for nonobservation of buffer ions in MALDI mass spectra of montmorillonite samples are currently unknown. However, a remarkable ability of the montmorillonite to neutralize salts and buffers encouraged us to test montmorillonite as a natural purifier for contaminated samples of RNA. In these experiments, homoionic $\mathrm{Li}-$, $\mathrm{Na}-$, and $\mathrm{K}$-enriched montmorillonites were mixed with a solution containing the oligonucleotides from ImpU. The solution itself (Sample P) produced a MALDI mass spectrum consisting of the buffer clusters peaks. The Sample P was applied to $\mathrm{Li}-, \mathrm{Na}-$, and $\mathrm{K}$-enriched montmorillonites producing Samples S (Scheme 1), whose mass spectra are shown on Figure 4. The difference between the signals was $\sim 309$ Da corresponding to the $\mathrm{pU}$ unit. The use of Li-montmorillonite resulted in a significant improvement of the mass spectrum (Figure 4c). Oligomers having up to at least 35 nucleotides could be easily accounted for. The quality of this mass spectrum was compared to that of the desalted solution, and the peaks were significantly better resolved than that obtained by mixing the reaction solution with either Na- (Figure 4b) or K-enriched (Figure 4a) montmorillonites. It was characterized by the detection of high mass products and good signal-to-noise ratio. Peak widths (at $50 \%$ of the height) for the oligonucleotides having $20 \mathrm{pU}$ units were $\sim 110, \sim 135$, and $\sim 165 \mathrm{Da}$ for the sample placed on Li-, Na-, and K-enriched montmorillonites, respectively. Li-enriched montmorillonite can therefore be used, so avoiding the purification of salt and buffer contaminated RNA and DNA samples.

Li-montmorillonite should be considered as a substitute for the more usual Na-containing montmorillonite as a catalyst for the oligomerization reaction. Its use would ensure the maximum sensitivity and the highest mass range of the subsequent direct MALDI analysis of the mineral on the assumption that the presence of $\mathrm{Li}^{+}$
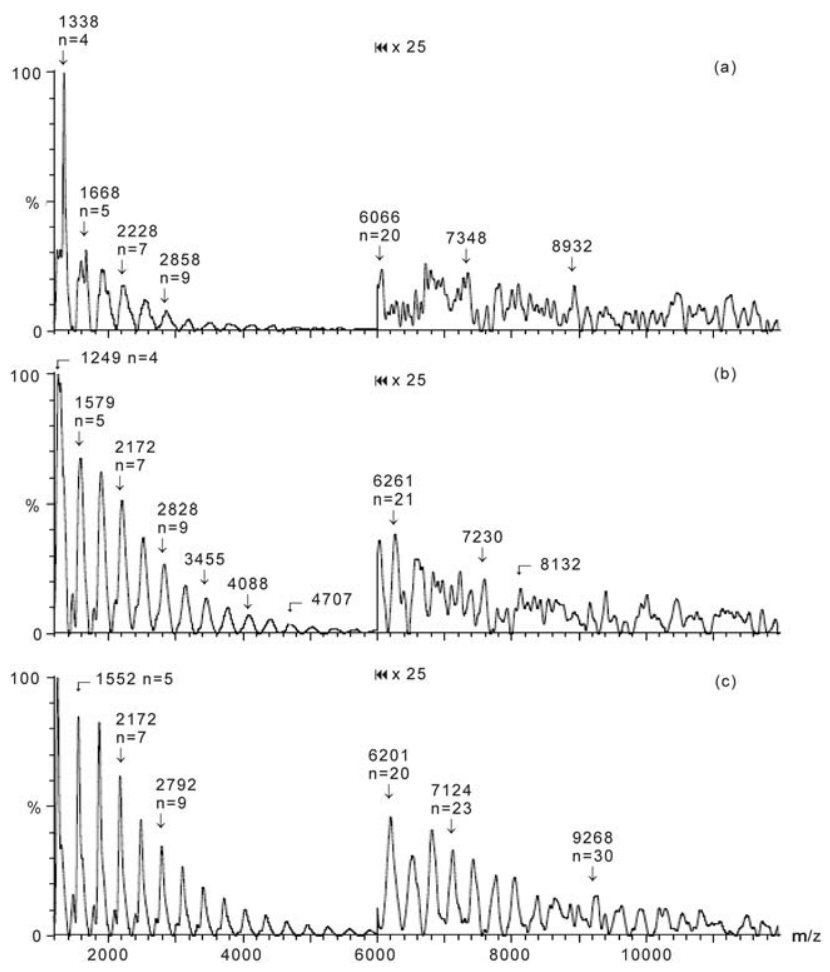

Figure 4. MALDI $(-)$ mass spectra of oligomerization products of ImpU: (a) Sample P mixed with K-montmorillonite, (b) sample $P$ mixed with Na-montmorillonite, (c) Sample $P$ mixed with Li-montmorillonite.

in the catalyst does not change the reaction pathway(s) leading to RNA oligomers.

The removal of salts and buffers is a standard and often a necessary procedure in DNA and RNA sample preparation for MALDI analysis [13]. The purpose of it is to remove alkali metals and other contaminants, the presence of which degrade the mass spectra. At the same time, alkali metals in small amounts can be useful in the molecular weight determination. It is well known that the stability of alkali-metal-containing ions is higher than their "desalted" analog [16, 17]. The presence of a metal atom(s) can therefore stabilize molecular ions of otherwise unstable gas-phase species.

\section{Conclusions}

The present study demonstrated that montmorillonite could be subjected to direct MALDI analysis for the analysis of DNA and RNA. Oligomers of various sizes were detected for adenosine and uracil derivatives. The procedure of sample preparation is similar to that for solutions and involves mixing with the corresponding matrix and ammonium salt. THAP and ATT were the matrixes allowing for the detection of oligomers having up to 40 bases. One of the major advantages of using montmorillonite is its high tolerance to salts and buffers. Oligonucleotides on the montmorillonite catalyst can be analyzed by MALDI without any purification. The procedure involving mixing solutions having high 
concentrations of salts and buffers with a (homoionic) montmorillonite can be considered as a method of sample cleanup. Whether or not it will find its place as an alternative to classical sample desalting, the use of montmorillonite does make it possible to analyze products of reactions where it is applied as a catalyst. Li-enriched montmorillonites have distinctive advantages over Na- and K-containing montmorillonites. The volatility of Li salts from the catalyst surface is comparable to that of desalted samples and significantly higher than their Na- and K-containing analogs. The size of oligomers detected after desalting (Samples R) and after placing Samples $\mathrm{P}$ on Li-montmorillonite (Samples S) were comparable and substantially exceeded those detected when the sample was placed on Na- and K-montmorillonites. Various experimental factors, including the ease of sample preparation, laser power, resolution and peak widths (in linear mode) and desalting abilities should be taken into account if montmorillonites are considered for use with samples other than those generated by reactions described in the present work.

\section{Acknowledgments}

The authors acknowledge the Department of Chemistry and Chemical Biology for continuing support of the Mass Spectrometry Facility. They gratefully acknowledge the National Science Foundation for the purchase of the MALDI-TOF instrument (NSF grant CHE-0078056). The research on origin of life is supported by NSF grant CHE-0413739 and NASA grant NAG5-12,750 that supports the NY Center for Studies of the Origin of Life.

\section{References}

1. Ferris, J. P. Mineral catalysis and prebiotic synthesis: Montmorillonitecatalyzed formation of RNA. Elements 2005, 1, 145-149.
2. Huang, W.; Ferris, J. P. Synthesis of 35-40 mers of RNA oligomers from unblocked monomers. A simple approach to the RNA world. Chem. Commun. 2003, 1458-1459.

3. Wang, K.-J.; Ferris, J. P. Catalysis and selectivity in prebiotic synthesis: Initiation of formation of oligo(U)s on montmorillonite clay by adenosine-5'-methylphosphate. Origins Life Evol. Biosphere 2005, 35, 187-212.

4. Ferris, J. P. Montmorillonite catalysis of 30-50 mer oligonucleotides: Laboratory demonstration of potential steps in the origin of the RNA world. Origins Life Evol. Biosphere 2002, 32, 311-332.

5. Ferris, J. P. The synthesis of the first polyribonucleotides: The role of catalysis. Chem. Tracts Biochem. Mol. Biol. 1999, 12, 419-431.

6. Rupert, J. P.; Granquist, W. T.; Pannavaia, T. J. Catalytic properties of clay minerals. Mineralogical Society Monograph. Chem. Clays Clay Miner. 1987, 6, 275-318.

7. Banin, A.; Lawless, J. G.; Mazzurco, J.; Church, F. M.; Margulies, L.; Orenberg, J. B. pH Profile of the adsorption of nucleotides onto montmorillonite. II. Adsorption and desorption of $5^{\prime}$-AMP in ironcalcium montmorillonite systems. Origins of Life 1985, 15, 89-101.

8. Joyce, G. F.; Inoue, T.; Orgel, L. E. Nonenzymatic template-directed synthesis on RNA random copolymers. $\operatorname{Poly}(\mathrm{C}, \mathrm{U})$ templates. J. Mol. Biol. 1984, 176, 279-306.

9. Zhu, Y. F.; Chung, C. N.; Taranenko, N. I.; Allman, S. L.; Martin, S. A.; Haff, L.; Chen, C. H. The study of 2,3,4-trihydroxyacetophenone and 2,4,6-trihydroxyacetophenone as matrixes for DNA detection an matrixassisted laser desorption/ionization time-of-flight mass spectrometry. Rapid Commun. Mass Spectrom. 1996, 10, 383-388.

10. Lecchi, P.; Le, H. M.; Pannell, L. K. 6-Aza-2-thiothymine: A matrix for MALDI spectra of oligonucleotides. Nucleic Acids Res. 1995, 23, 12761277.

11. Wu, K. J.; Stedding, A.; Becker, C. H. Matrix-assisted laser desorption time-of-flight mass spectrometry of oligonucleotides using 3-hydroxypicolinic acid as an ultraviolet-sensitive matrix. Rapid Commun. Mass Spectrom. 1993, 7, 142-146.

12. Streletskii, A. V.; Kozlova, A. Yu.; Esipov, D. S.; Kayushin, A. L.; Korosteleva, M. D.; Esipov, S. E. Determination of oligonucleotide molecular masses by MALDI mass spectrometry. Russ. J. Bioorg. Chem. 2005, 31, 139-145.

13. Nordhoff, E.; Kirpekar, F.; Roepstorff, P. Mass spectrometry of nucleic acids. Mass Spectrom Rev. 1996, 15, 67-138.

14. Dass, C. Characterization of oligonucleotides. In Principles and Practice of Biological Mass Spectrometry; Wiley: New York, 2001; Chap XV, pp 346-373.

15. Simmons, T. A.; Green-Church, K. B.; Limbach, P. A. Nucleic acids and nucleotides studied using mass spectrometry. In Encyclopedia of Spectroscopy and Spectrometry, Applications Vol. II; Lindon, J.; Tranter, G.; Holmes, J., Eds.; Academic Press: London, 1999; pp 1681-1688.

16. Ngoka, L. C.; Gal, J.-F.; Lebrilla, C. B. Effect of cations and charge types on the metastable decay rates of oligosaccharides. Anal. Chem. 1994, 66, 692-698.

17. Cancilla, M. T.; Penn, S. G.; Carroll, J. A.; Lebrilla, C. B. Coordination of alkali metals to oligosaccharides dictates fragmentation behavior in matrix assisted laser desorption ionization/Fourier transform mass spectrometry. J. Am. Chem. Soc. 1996, 118, 6736-6745. 